\title{
A new health classification scheme based on fuzzy hesitant information
}

\author{
J. C. R. Alcantud, ${ }^{1}$ R. de Andrés Calle, ${ }^{1}$ T. González-Arteaga ${ }^{2}$ \\ ${ }^{1}$ Facultad de Economía y Empresa and Multidisciplinary Institute of Enterprise (IME), \\ Universidad de Salamanca, Spain \\ ${ }^{2}$ Facultad de Ciencias, Universidad de Valladolid, Spain
}

\begin{abstract}
The evaluation of the health status of a population is a key question in Health Policy. The measurement of the health status is a relevant process to keep and improve the countries' level of economic development. In spite of this relevance, the current evaluation methodologies are not sufficiently well-defined either designed due to the fact that health is an inherently subjective notion. In order to overcome these drawbacks, first of all we propose a newer way to manage such uncertainty by means of the Fuzzy Logic Theory. Regarding the methodology, we develop a new approach to classify populations according to their health status based on hesitant fuzzy information. Additionally, we apply the proposed mechanism on real health data from 15 European countries.
\end{abstract}

Keywords: Health measurement, Hesitant information, Geometric score, Geometric variance.

\section{Introduction}

The measurement of health is central to the evaluation of health care and its policy. In consequence, the estimation of the health status of a country or a group of individuals is the basis of an extensive area of health-related studies.

The definition of health and its measurement are controversial issues. The World Health Organization (WHO) describes health as a "state of complete physical, mental,and social well-being and not merely the absence of disease or infirmity" and establishes the following five reasons for its study (see [1], [2], [3], [4], among others) :

- Health status helps to measure the efficiency and effectiveness of medical interventions.

- Evaluation of the quality of care. The evaluations of medical care in terms of patient outcomes include health status.

- Health status estimates the needs of a population. Its measure is a useful tool for describing population health levels.

- Health state improves clinical decisions.
- Health changes over time and associations between health and other variables can be studied by means of health status.

Taking into account the previous arguments, the measurement of the health status has been considered from different approaches. Ware et al. establish in [1] that a "good" health measurement should be practical, reliable, valid and objective. From other point of view, Hausman [4] declares that health measures must be at least intervally significant and interpersonally comparable.

Traditionally, the measure of the health state has been analyzed from an objective perspective by means of biochemical, physiological and anatomical indexes. However, the health status has recently been considered as a multi-dimensional concept which can be measured from different points of view (see [5],[6], [7], [8] and [9], among other). Some of these aspects may be measured by means of clinical test but others need to be measured taking into account the perceptions that patients have on them. Taking into account such points, the researchers have carried out different measurement of health status based on the respondent's assessment of their own health status, the so-called selfassessed health (SAH). To be precise, this information is usually obtained from a question such as: "In your opinion, how is your own health state today?", where individuals must select one among five possible states, typically ranking from "very good or excellent" to "very bad or very poor".

With the purpose of managing the uncertainty associated with available data we propose a new methology to classify populations according to their health status. We rely on the capabilities of Fuzzy logic Theory as a very successful tool to manage human subjectivity, uncertainty and hesitancy in decision-making contexts (see [10], [11] and [12] for example).

As early as Zadeh [13] recognized that imprecise human knowledge (and especially group knowledge) cannot be suitably represented by fuzzy sets, and that some generalizations are called for. In this paper we are interested in the practical implementation of a new methodology that permits to rank hesitant fuzzy elements, which are the fundamental constituent of hesitant fuzzy sets (cf., Torra [14], originally considered by Grattan-Guinness [15]). 
The motivation for using this concept in decision making is clearly explained e.g., in Xu [16]. This reference justifies that hesitant fuzzy elements and sets have produced an extensive theoretical and applied literature. Some fundamental issues about hesitant fuzzy sets are gathered in Rodríguez et al. [17].

Availabla data from reputed sources permit to attach a hesitant fuzzy element with each country on a single attribute (health). Our innovative methodology for ranking hesitant fuzzy elements provides a classification of countries by their health status. We follow the recent trend based on scoredeviation algorithms (Chen et al. [18] and Liao and $\mathrm{Xu}[19]$ ), which have been applied e.g., to evaluate the service quality of airlines in [19]. Inspired by the arguments in favour of the geometric mean in the computation of indexes in social sciences (cf., Herrero et al. [20] and the references therein) we propose the geometric score to rank hesitant fuzzy elements. As a tie-breaking mechanism, the natural concept of a geometric variance is introduced.

This paper is organized as follows. Section 2 establishes some basic notation and definitions. Moreover, we introduce a newer hesitant fuzzy element score and a novel associated variance indicator. In Section 3 we present our proposal for attaching hesitant fuzzy elements to populations attending to the health attribute, as well as our scheme for classifying them. In Section 4 we put in practice our methodology that permits to rank the populations of 15 European countries attending to their health state. We conclude in Section 5.

\section{Notation and definitions}

For any set $A, \mathcal{F}^{*}(A)$ denotes the set of non-empty finite subsets of $A$.

\subsection{HFEs and related notions}

Definition 1 (Xia and $\mathrm{Xu}$ [21]) A hesitant fuzzy element (HFE) is a non-empty, finite subset of $[0,1]$. The set of HFEs is denoted by $\mathcal{F}^{*}([0,1])$.

The motivation for using this concept in decision making is clearly explained e.g., in $\mathrm{Xu}$ [16, Chapter 1.1]. The basic idea is that people are sometimes hesitant or irresolute, and a handful of possible membership values should be considered e.g., because each one has been defended by an advisor. This does not imply any convexity or intervalproperty of the degrees with which the alternatives satisfy an attribute. In this way HFEs, which are the fundamental constituent of hesitant fuzzy sets or HFSs (cf., Torra [14]), have produced an intense literature that separates from e.g., intuitionistic fuzzy sets and interval-valued fuzzy sets.

By restricting ourselves to $\mathcal{F}^{*}([0,1])$ we require the extremely mild condition that on each alternative, at least one assessment must be made.
For each hesitant fuzzy element $h$, we can arrange its elements so that we denote

$$
h=\left\{h_{1}, \ldots, h_{l_{h}}\right\}
$$

with $h_{1}<\ldots<h_{l_{h}}$. In particular, the cardinality of the HFE $h$ is $l_{h}=|h|$. Observe that because $h$ is a set, repetitions are excluded by definition.

\subsection{On ranking orders of HFEs}

In the context of multi-person decision making, when the opinions are collected according to either the model by HFEs (one single attribute) or the richer model by HFSs (multiple attributes) the decision maker needs to reach a decision that considers all that information.

In this subsection we refer to the problem of comparing and ranking HFEs. To that purpose the following notion may be useful.

Definition $2 A$ score on HFEs is a mapping $s: \mathcal{F}^{*}([0,1]) \longrightarrow[0,1]$ that verifies the equivalence: $s\left(h=\left\{h^{1}, \ldots, h^{l_{h}}\right)\right\}=1$ if and only if $h=\{1\}$.

The literature has provided some examples of scores on HFEs that have been subsequently applied for decision making purposes, as explained in the up-to-date references Rodríguez et al. [17], Xu [16] or Zhang and Xu [22] among others. Thus for example, for each typical hesitant fuzzy set $h=\left\{h_{1}, \ldots, h_{l_{h}}\right\}$ :

a. Xia and $\mathrm{Xu}[21]$ define the score

$$
\mathcal{S}_{1}(h)=\frac{h_{1}+\ldots+h_{l_{h}}}{l_{h}}
$$

b. Farhadinia [23] proposes to start with a monotone non-decreasing sequence $\{\delta(1), \ldots, \delta(n), \ldots\}$ of positive numbers and then use the score

$$
\mathcal{S}_{2}(h)=\frac{\delta(1) h_{1}+\ldots+\delta\left(l_{h}\right) h_{l_{h}}}{\delta(1)+\ldots+\delta\left(l_{h}\right)}
$$

This includes the previous case when the sequence is $\{1,1, \ldots, 1, \ldots\}$.

Scores are useful since they permit to rank HFEs in an immediate manner: the higher the score of an HFE, the better it is considered. Of course, ties among different HFEs arise on a regular basis. In order to avoid this undesirable situation Chen et al. [24] and Liao and Xu [19] propose respective methodologies to distinguish among HFEs that are scored the same according to Xia and Xu's [21] score. Their idea is that when $\mathcal{S}_{1}(h)=\mathcal{S}_{1}\left(h^{\prime}\right)$, this tie is broken by appealing to the deviation degree of $h$ and $h^{\prime}$, either in statistical terms $\left(\sigma_{1}\right)$ or among all pairs of elements in the HFEs $\left(\sigma_{2}\right)$, respectively. These deviation degrees are defined as follows:

$\sigma_{1}\left(h=\left\{h_{1}, \ldots, h_{l_{h}}\right\}\right)=\left(\frac{1}{l_{h}} \sum_{i=1, \ldots, l_{h}}\left(h_{i}-\mathcal{S}_{1}(h)\right)^{2}\right)^{\frac{1}{2}}$ 
and

$\sigma_{2}\left(h=\left\{h_{1}, \ldots, h_{l_{h}}\right\}\right)=\left(\frac{1}{l_{h}} \sum_{i, j=1, \ldots, l_{h}}\left(h_{i}-h_{j}\right)^{2}\right)^{\frac{1}{2}}$

We can note that there is a simple relationship between $\sigma_{1}$ and $\sigma_{2}$ concretely, $\frac{\sigma_{2}^{2}}{\sigma_{1}^{2}}=2 n^{2}$.

Here we adopt the latter methodology (based on tie-breaking procedures for HFEs with equal evaluations). Therefore for the purpose of illustration and comparison, we recall that Chen et al. [24] present the following procedure:

Definition 3 Let $h$ and $h^{\prime}$ be two HFEs on $X$. We declare:

1. If $\mathcal{S}_{1}(h)<\mathcal{S}_{1}\left(h^{\prime}\right)$ then $h^{\prime}$ is superior to $h$.

2. If $\mathcal{S}_{1}(h)=\mathcal{S}_{1}\left(h^{\prime}\right)$ then:

- If $\sigma_{1}(h)<\sigma_{1}\left(h^{\prime}\right)$ then $h$ is superior to $h^{\prime}$.

- If $\sigma_{1}(h)>\sigma_{1}\left(h^{\prime}\right)$ then $h^{\prime}$ is superior to $h$.

- If $\sigma_{1}(h)=\sigma_{1}\left(h^{\prime}\right)$ then $h$ is equivalent to $h^{\prime}$.

And similarly, Liao and Xu [19] advocate for using a related proposal:

Definition 4 Let $h$ and $h^{\prime}$ be two HFEs on $X$. We declare:

1. If $\mathcal{S}_{1}(h)<\mathcal{S}_{1}\left(h^{\prime}\right)$ then $h^{\prime}$ is superior to $h$.

2. If $\mathcal{S}_{1}(h)=\mathcal{S}_{1}\left(h^{\prime}\right)$ then:

- If $\sigma_{2}(h)<\sigma_{2}\left(h^{\prime}\right)$ then $h$ is superior to $h^{\prime}$.

- If $\sigma_{2}(h)>\sigma_{2}\left(h^{\prime}\right)$ then $h^{\prime}$ is superior to $h$.

- If $\sigma_{2}(h)=\sigma_{2}\left(h^{\prime}\right)$ then $h$ is equivalent to $h^{\prime}$.

\section{A new score-variance mechanism for hesitant fuzzy elements}

As is apparent from the discussion above, in order to implement any score-variance based model to rank HFEs two items are needed. The first one is an aggregative indicator like $\mathcal{S}_{1}$. And in case of a tie, another parameter intervenes and prioritizes the HFEs by their dispersion (from smaller to higher).

We proceed to make new proposals for these two steps.

As an aggregative indicator we propose to use a geometric score rather than an arithmetic or statistical mean, which is the nature of $\mathcal{S}_{1}$. We believe that it is sensible to discard the use of arithmetic means because reputed scholars as Chakravarty [25], Foster et al. [26], Herrero et al. [27], Seth [28], among others, claim that there are at least three sources of discomfort when this mean is applied in order to aggregate information. And then they give arguments that justify that the geometric mean is a much better choice due to its properties.

Thus following the convincing arguments by Herrero et al. [20] in their design of a newer human development index, we introduce a new geometric score for HFEs:

Definition 5 The geometric score on HFEs is the mapping $s: \mathcal{F}^{*}([0,1]) \longrightarrow[0,1]$ such that

$$
\mathcal{S}_{G}\left(h=\left\{h_{1}, \ldots, h_{l_{h}}\right\}\right)=\left(h_{1} \cdots h_{l_{h}}\right)^{\frac{1}{l_{h}}} .
$$

This geometric score penalizes the dispersion of the values under consideration. Nevertheless this score does not necessarily discriminate between different HFEs, as was the case of the previously mentioned scores. Therefore in the second place we propose to appeal to the following new concept in order to break ties:

Definition 6 The geometric variance on HFEs is defined by

$$
\begin{gathered}
\mathcal{V}_{G}\left(h=\left\{h_{1}, \ldots, h_{l_{h}}\right\}\right)= \\
=\exp \left(\frac{1}{l_{h}} \sum_{i=1, \ldots, l_{h}}\left(\log \left(h_{i}\right)-\mathcal{S}_{1}(\log (h))\right)^{2}\right)
\end{gathered}
$$

where $\log (h)=\left(\log \left(h_{1}\right), \ldots, \log \left(h_{l_{h}}\right)\right)$.

The motivation for using this expression is the following. The logarithm of $\mathcal{S}_{G}(h)$ is simply $\mathcal{S}_{1}(\log (h))$ because

$$
\begin{aligned}
& \log \mathcal{S}_{G}\left(\left\{h_{1}, \ldots, h_{l_{h}}\right\}\right)=\log \left(h_{1} \cdots h_{l_{h}}\right)^{\frac{1}{l_{h}}}= \\
= & \frac{1}{l_{h}}\left(\log \left(h_{1}\right)+\ldots+\log \left(h_{l_{h}}\right)\right)=\mathcal{S}_{1}(\log (h)) .
\end{aligned}
$$

This is to say, $\mathcal{S}_{G}(h)=\exp \left(\mathcal{S}_{1}(\log (h))\right)$ : the geometric mean is the exponential of the arithmetic mean of the logarithms of the variables. It is therefore natural to define $\mathcal{V}_{G}(h)$ as above by replicating the argument: the geometric variance is the exponential of the statistical variance of the logarithms of the variables.

In conclusion, based on the newly defined $\mathcal{S}_{G}$ and $\mathcal{V}_{G}$ we propose a newer score-variance model to rank HFEs as follows:

Definition 7 Let $h$ and $h^{\prime}$ be two HFEs on $X$. We declare:

1. If $\mathcal{S}_{G}(h)<\mathcal{S}_{G}\left(h^{\prime}\right)$ then $h<h^{\prime}$.

2. If $\mathcal{S}_{G}(h)=\mathcal{S}_{G}\left(h^{\prime}\right)$ then:

- If $\mathcal{V}_{G}(h)<\mathcal{V}_{G}\left(h^{\prime}\right)$ then $h$ is superior to $h^{\prime}$.

- If $\mathcal{V}_{G}(h)>\mathcal{V}_{G}\left(h^{\prime}\right)$ then $h^{\prime}$ is superior to $h$. 
- If $\mathcal{V}_{G}(h)=\mathcal{V}_{G}\left(h^{\prime}\right)$ then $h$ is equivalent to $h^{\prime}$.

Table 1 summarizes various approaches to rank HFEs.

\begin{tabular}{ll} 
Ranking approach & Authors \\
\hline $\mathcal{S}_{1}$ score & Xia and Xu [21] \\
$\mathcal{S}_{2}$ score & Farhadinia $[23]$ \\
$\mathcal{S}_{1}$ score and $\sigma_{1}:$ Def. 3 & Chen et al. $[24]$ \\
$\mathcal{S}_{1}$ score and $\sigma_{2}:$ Def. 4 & Liao and Xu [19] \\
$\mathcal{S}_{G}$ score and $\mathcal{V}_{G}:$ Def. 7 & Our proposed approach \\
\hline
\end{tabular}

Table 1: Review of ranking orders of HFEs

We stress the fact that our proposal incorporates Xia and Xu's [21] score (within the construction of $\mathcal{V}_{G}$ ) as well as a new score and a new tie-breaking indicator. This supposes a richer approach in the analysis of ranking HFEs.

\section{A classification of countries by health status based on hesitant information: empirical application to European countries}

In this Section we exemplify the use of the aforementioned methodology for ranking a sample of 15 European countries attending to their self-perceived health status.

\subsection{Real exemplifications of our new ranking procedure for HFEs}

As mentioned above, the appropiate way to make comparisons based on health information is a source of dispute (see [29] for a fully-fledged discussion). We contribute to this debate by the recourse to Definition 7 or any other ranking procedure in Table 1.

In this contribution, we use the evaluations of health status of 15 European countries provided by three agencies (the experts), namely, the Organization for economic co-operation and development (OECD), the Statistical office of the European Union (Eurostat) and the Spanish statistical office (INE). Traditionally, these agencies classify the health status of the population in five different categories: "very good", "good", "fair", "bad" or "very bad". Moreover, the population is arrange in classes attending to age, gender and other demographic characteristics

In this real example we focus on studying the overall perceived health status of female and male without other demographic subject. In this way every population -female and male- in the study is associated with a three-element HFE.

We now present an application of the proposed score-variance mechanism to the 2011 self-assessed health data of 15 European countries. The original data collected from the OECD, Eurostat and INE are shown in Table 2 and Table 3 for males and females, respectively. We use the aforementioned data in order to attach a HFE with each country following the procedure displayed in Figure 1. The results are shown in Table 4 and Table 5 .

Table 2: Evaluations of perceived male health status for the 15 countries made by the 3 agencies (year 2011)

\begin{tabular}{llll} 
Country & OECD & Eurostat & INE \\
\hline Austria & 0.731 & 0.730 & 0.731 \\
Belgium & 0.778 & 0.791 & 0.779 \\
Denmark & 0.778 & 0.784 & 0.776 \\
Finland & 0.693 & 0.440 & 0.691 \\
France & 0.721 & 0.720 & 0.723 \\
Germany & 0.631 & 0.635 & 0.635 \\
Greece & 0.798 & 0.806 & 0.799 \\
Ireland & 0.843 & 0.844 & 0.844 \\
Italy & 0.608 & 0.609 & 0.608 \\
Luxembourg & 0.762 & 0.768 & 0.762 \\
Netherlands & 0.798 & 0.800 & 0.800 \\
Portugal & 0.532 & 0.570 & 0.533 \\
Spain & 0.712 & 0.732 & 0.713 \\
Sweden & 0.785 & 0.375 & 0.786 \\
United Kingdom & 0.780 & 0.351 & 0.781 \\
\hline
\end{tabular}

Table 3: Evaluations of perceived female health status for the 15 countries made by the 3 agencies (year 2011)

\begin{tabular}{llll} 
Country & OECD & Eurostat & INE \\
\hline Austria & 0.710 & 0.709 & 0.709 \\
Belgium & 0.708 & 0.729 & 0.709 \\
Denmark & 0.722 & 0.735 & 0.726 \\
Finland & 0.682 & 0.441 & 0.683 \\
France & 0.666 & 0.667 & 0.668 \\
Germany & 0.576 & 0.577 & 0.578 \\
Greece & 0.738 & 0.761 & 0.740 \\
Ireland & 0.820 & 0.822 & 0.819 \\
Italy & 0.531 & 0.534 & 0.532 \\
Luxembourg & 0.723 & 0.724 & 0.723 \\
Netherlands & 0.740 & 0.740 & 0.740 \\
Portugal & 0.432 & 0.513 & 0.434 \\
Spain & 0.646 & 0.676 & 0.648 \\
Sweden & 0.753 & 0.336 & 0.735 \\
United Kingdom & 0.735 & 0.321 & 0.753 \\
\hline
\end{tabular}

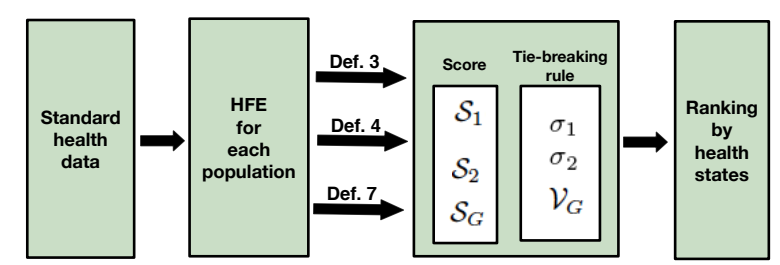

Figure 1: Health classification scheme 


\begin{tabular}{ll} 
Countries & Health HFEs \\
\hline Austria & $(0.731,0.730,0.731)$ \\
Belgium & $(0.778,0.791,0.779)$ \\
Denmark & $(0.778,0.784,0.776)$ \\
Finland & $(0.693,0.440,0.691)$ \\
France & $(0.721,0.720,0.723)$ \\
Germany & $(0.631,0.635,0.635)$ \\
Greece & $(0.798,0.806,0.799)$ \\
Ireland & $(0.843,0.844,0.844)$ \\
Italy & $(0.608,0.609,0.608)$ \\
Luxembourg & $(0.762,0.768,0.762)$ \\
Netherlands & $(0.798,0.800,0.800)$ \\
Portugal & $(0.532,0.570,0.533)$ \\
Spain & $(0.712,0.732,0.713)$ \\
Sweden & $(0.785,0.375,0.786)$ \\
United Kingdom & $(0.780,0.351,0.781)$ \\
\hline
\end{tabular}

Table 4: HFEs capturing perceived male health status by countries.

\begin{tabular}{ll} 
Countries & Health HFEs \\
\hline Austria & $(0.710,0.709,0.709)$ \\
Belgium & $(0.708,0.729,0.709)$ \\
Denmark & $(0.722,0.735,0.726)$ \\
Finland & $(0.682,0.441,0.683)$ \\
France & $(0.666,0.667,0.668)$ \\
Germany & $(0.576,0.577,0.578)$ \\
Greece & $(0.738,0.761,0.740)$ \\
Ireland & $(0.820,0.822,0.819)$ \\
Italy & $(0.531,0.534,0.532)$ \\
Luxembourg & $(0.723,0.724,0.723)$ \\
Netherlands & $(0.740,0.740,0.740)$ \\
Portugal & $(0.432,0.513,0.434)$ \\
Spain & $(0.646,0.676,0.648)$ \\
Sweden & $(0.753,0.336,0.735)$ \\
United Kingdom & $(0.735,0.321,0.753)$ \\
\hline
\end{tabular}

Table 5: HFEs capturing perceived female health status by countries.

In order to implement our methodology (cf., Definition 7) we need to calculate geometric scores associated with the HFEs in Table 4 and Table 5 . These figures are given in Table 6 and Table 7 for males and females, respectively. The corresponding classifications of the countries attending to their health status are compiled in Tables 8 and 9 . The display of these rankings can be observed in Figure 2.

\subsection{Comparison with other existing methodologies}

For the sake of the comparison, we now present the conclusions of the analogous analysis under the positions of $\mathcal{S}_{2}$ and Definitions 3 and 4 that include tie-breaking procedures. To that purpose we need to compute $\mathcal{S}_{2}, \mathcal{S}_{1}, \sigma_{1}$, and $\sigma_{2}$ associated with the HFEs in Table 4 and Table 5 . These results are

\begin{tabular}{ll} 
Countries & Health index \\
\hline Austria & 0.733 \\
Belgium & 0.785 \\
Denmark & 0.781 \\
Finland & 0.598 \\
France & 0.724 \\
Germany & 0.638 \\
Greece & 0.803 \\
Ireland & 0.845 \\
Italy & 0.611 \\
Luxembourg & 0.766 \\
Netherlands & 0.801 \\
Portugal & 0.548 \\
Spain & 0.721 \\
Sweden & 0.617 \\
United Kingdom & 0.601
\end{tabular}

Table 6: Male health index based on geometric score.

\begin{tabular}{ll} 
Countries & Health index \\
\hline Austria & 0.709 \\
Belgium & 0.715 \\
Denmark & 0.728 \\
Finland & 0.590 \\
France & 0.667 \\
Germany & 0.577 \\
Greece & 0.749 \\
Ireland & 0.820 \\
Italy & 0.532 \\
Luxembourg & 0.723 \\
Netherlands & 0.740 \\
Portugal & 0.458 \\
Spain & 0.657 \\
Sweden & 0.571 \\
United Kingdom & 0.562 \\
\hline
\end{tabular}

Table 7: Female health index based on geometric score.

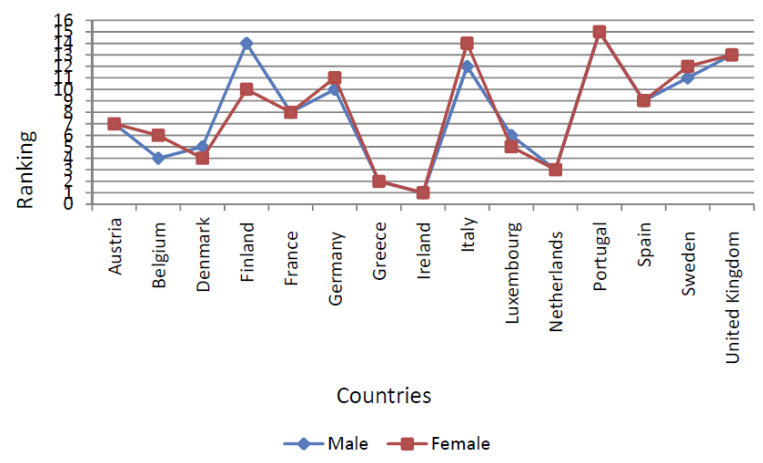

Figure 2: Comparative study of classifications for males and females attending to our proposal

given in Tables 10, 11, 12 and 13, respectively.

We can observe in Table 10 that there is a tie between Italy and Finland with $\mathcal{S}_{2}=0,608$. This 


\begin{tabular}{ll} 
Countries & $\mathcal{S}_{G}$ \\
\hline Ireland & 0.845 \\
Greece & 0.803 \\
Netherlands & 0.801 \\
Belgium & 0.785 \\
Denmark & 0.781 \\
Luxembourg & 0.766 \\
Austria & 0.733 \\
France & 0.724 \\
Spain & 0.721 \\
Germany & 0.638 \\
Sweden & 0.617 \\
Italy & 0.611 \\
United Kingdom & 0.601 \\
Finland & 0.598 \\
Portugal & 0.548 \\
\hline
\end{tabular}

Table 8: Classification by male health states according to our proposal.

\begin{tabular}{ll} 
Countries & $\mathcal{S}_{G}$ \\
\hline Ireland & 0.820 \\
Greece & 0.746 \\
Netherlands & 0.740 \\
Denmark & 0.728 \\
Luxembourg & 0.723 \\
Belgium & 0.715 \\
Austria & 0.709 \\
France & 0.667 \\
Spain & 0.657 \\
Finland & 0.590 \\
Germany & 0.577 \\
Sweden & 0.571 \\
United Kingdom & 0.562 \\
Italy & 0.532 \\
Portugal & 0.458 \\
\hline
\end{tabular}

Table 9: Classification by female health states according to our proposal.

mechanism does not provide tie-breaking rule, it does not take into account the situation that two HFEs have the same score. Consequently, this approach is not sufficiently discriminatory and it loses information.

Moreover, we note that Definitions 3 and 4 provide exactly the same classification for male population, but it is different from the conclusion in Table 8 according to the methodology provided here (see Figure 3). In addition, there is not any tie among countries for female populations (see Table 9, 11 and 13). The different methodologies do not produce the exactly the same classification (see Figure 4).

\section{Conclusion}

In this paper we give a new methodology for ranking hesitant fuzzy elements based on the geomet-

\begin{tabular}{ll} 
Countries & $\mathcal{S}_{2}$ \\
\hline Ireland & 0.844 \\
Greece & 0.801 \\
Netherlands & 0.800 \\
Belgium & 0.783 \\
Denmark & 0.779 \\
Luxembourg & 0.764 \\
Austria & 0.731 \\
France & 0.722 \\
Spain & 0.719 \\
Sweden & 0.649 \\
United Kingdom & 0.638 \\
Germany & 0.635 \\
Finland & 0.608 \\
Italy & 0.608 \\
Portugal & 0.545 \\
\hline
\end{tabular}

Table 10: Classification by male health states according to Farhadinia's score

\begin{tabular}{ll} 
Countries & $\mathcal{S}_{2}$ \\
\hline Ireland & 0.820 \\
Greece & 0.747 \\
Netherlands & 0.740 \\
Denmark & 0.728 \\
Luxembourg & 0.723 \\
Belgium & 0.716 \\
Austria & 0.709 \\
France & 0.667 \\
Spain & 0.657 \\
United Kingdom & 0.606 \\
Sweden & 0.605 \\
Finland & 0.602 \\
Germany & 0.577 \\
Italy & 0.533 \\
Portugal & 0.460 \\
\hline
\end{tabular}

Table 11: Classification by female health states according to Farhadinia's score

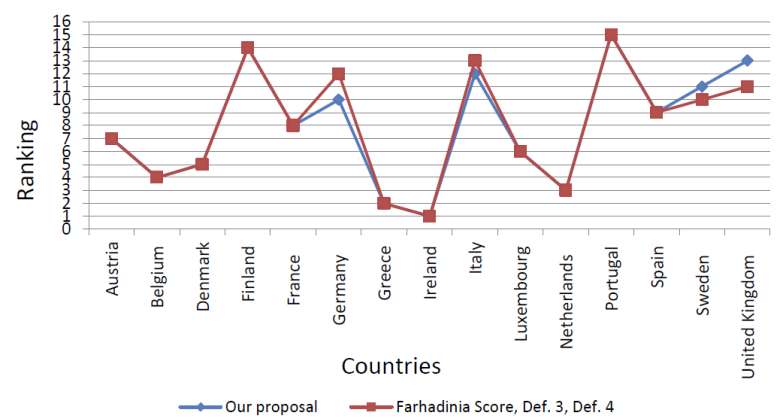

Figure 3: Comparative study of classifications by male health states

ric mean and variance. We give a procedure by which the real data of countries' self-assessed health naturally deliver hesitant fuzzy elements for each popu-lation in the analysis. Thus coupling both 


\begin{tabular}{llll} 
Countries & $\mathcal{S}_{1}$ & $\sigma_{1}$ & $\sigma_{2}$ \\
\hline Ireland & 0.835 & & \\
Greece & 0.793 & & \\
Netherlands & 0.791 & & \\
Belgium & 0.775 & & \\
Denmark & 0.772 & & \\
Luxembourg & 0.756 & & \\
Austria & 0.723 & & \\
France & 0.714 & & \\
Spain & 0.712 & & \\
Sweden & 0.642 & & \\
United Kingdom & 0.631 & & \\
Germany & 0.629 & & \\
Italy & 0.602 & 0,0005 & 0,008 \\
Finland & 0.602 & 0,1188 & 0,2058 \\
Portugal & 0.540 & & \\
\hline
\end{tabular}

Table 12: Classification by male health states according to Definitions 3 and 4 .

\begin{tabular}{ll} 
Countries & $\mathcal{S}_{1}$ \\
\hline Ireland & 0.820 \\
Greece & 0.746 \\
Netherlands & 0.740 \\
Denmark & 0.728 \\
Luxembourg & 0.723 \\
Belgium & 0.715 \\
Austria & 0.709 \\
France & 0.667 \\
Spain & 0.657 \\
Sweden & 0.608 \\
United Kingdom & 0.603 \\
Finland & 0.602 \\
Germany & 0.577 \\
Italy & 0.532 \\
Portugal & 0.460 \\
\hline
\end{tabular}

Table 13: Classification by female health states according to Xia and Xu's score.

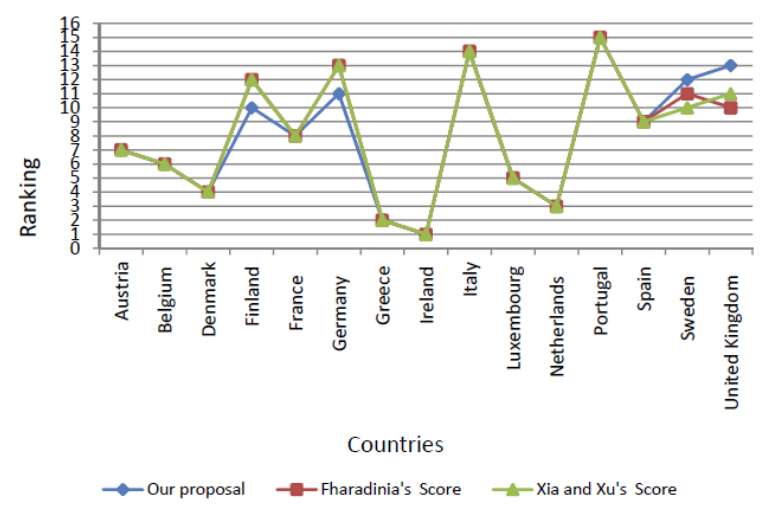

Figure 4: Comparative study of classifications by female health states

stages, ranking procedures for hesitant fuzzy elements can be used to compare health across popu- lations.

Finally, we believe that our real application will permit the scholars to implement future developments in health-related studies.

\section{Acknowledgment}

Financial support by the Spanish Ministerio de Ciencia e Innovación under Project ECO201231933 (J. C. R. Alcantud), Project ECO201232178, Project CGL2008-06003-C03-03/CLI (R. de Andrés Calle), and Project ECO2012-32178 (T. González-Arteaga) is gratefully acknowledged.

\section{References}

[1] J. E. Ware, R. H. Brook, A. R. Davies, and K. N. Lohr. Choosing measures of health status for individuals in general populations. American Journal of Public Health, 71:620-625, 1981.

[2] P. Rao and G. Sastry. Measurement of trends in health status. Technical report, World Health Organitation, 1990.

[3] R. Andrew Allison and J. E. Foster. Measuring health inequality using qualitative data. Journal of Health Economics, 23(3):505 - 524, 2004.

[4] D. M. Hausman. Valuing health: a new proposal. Health Economics, 19(3):280-296, 2010.

[5] EuroQol. A new facility for the measurement of health-related quality of life. Health Policy, 16:199-208, 1990.

[6] N. D. Beaulieu. Quality information and consumer health plan choices. Journal of Health Economics, 21:43-63, 2002.

[7] EuroQol. Measuring self reported population health: An International perspective based on EQ-5D. SpringMed Publishing, 2004.

[8] EuroQol. The measurement and valuation of health status using EQ-5D: A European perspective. Kluwer Academic Publisher, 2005.

[9] EuroQol. EQ-5D value sets: Inventory, comparative review and user guide. Springer, 2006.

[10] T. Tanino. Fuzzy preference orderings in group decision making. Fuzzy Sets and Systems, 12(2):117-131, 1984.

[11] J.C. Fodor and M. Roubens. Fuzzy preference modelling and multicriteria decision support. Kluwer Academic Publishers, 1994.

[12] J. Kacprzyk and H. Nurmi. Group decision making under fuzziness. In R. Slowinski, editor, Fuzzy sets in Decision Analysis, Operations research and Statistics. Springer, 1998.

[13] L. Zadeh. Outline of a new approach to analysis of complex systems and decision processes. IEEE Transactions on Systems, Man, and Cybernetics, 3:28-44, 1973.

[14] V. Torra. Hesitant fuzzy sets. International Journal of Intelligent Systems, 25(6):529-539, 2010 . 
[15] I. Grattan-Guinness. Fuzzy membership mapped onto intervals and many-valued quantities. Mathematical Logic Quarterly, 22:149160, 1976.

[16] Z. Xu. Hesitant Fuzzy Sets Theory, volume 314 of Studies in Fuzziness and Soft Computing. Springer International Publishing, 2014.

[17] R.M. Rodríguez, L. Martínez, V. Torra, Z.S. $\mathrm{Xu}$, and $\mathrm{F}$. Herrera. Hesitant fuzzy sets: state of the art and future directions. International Journal of Intelligent Systems, 29:495$524,2014$.

[18] H. Chen, A. H. I. Lee, and Y. Tong. Prioritization and operations NPD mix in a network with strategic partners under uncertainty. Expert Systems with Applications, 33(2):337 $346,2007$.

[19] H. Liao and Z. Xu. Satisfaction degree based interactive decision making under hesitant fuzzy environment with incomplete weights. International Journal of Uncertainty, Fuzziness and Knowledge-Based Systems, 22(04):553-572, 2014.

[20] C. Herrero, R. Martínez, and A. Villar. A newer human development index. Journal of Human Development and Capabilities: A Multi-Disciplinary Journal for PeopleCentered Development, 13(2):247-268, 2012.

[21] M. Xia and Z. Xu. Hesitant fuzzy information aggregation in decision making. International Journal of Approximate Reasoning, 52:395-407, 2011.

[22] X. Zhang and Z. Xu. The TODIM analysis approach based on novel measured functions under hesitant fuzzy environment. KnowledgeBased Systems, 61:48 - 58, 2014.

[23] B. Farhadinia. A novel method of ranking hesitant fuzzy values for multiple attribute decision-making problems. International Journal of Intelligent Systems, 28:752-767, 2013.

[24] N. Chen, Z. Xu, and M. Xia. Correlation coefficients of hesitant fuzzy sets and their applications to clustering analysis. Applied Mathematical Modelling, 37(4):2197 - 2211, 2013.

[25] S. R. Chakravarty. A generalized human development index. Review of Development Economics, 7(1):99-114, 2003.

[26] J. E. Foster. Measuring the distribution of human development: Methodology and an application to mexico. Journal of Human Development and Capabilities, 6(1):5-25, 2005.

[27] C. Herrero, R. Martínez, and A. Villar. Multidimensional social evaluation: An application to the measurement of human development. Review of Income and Wealth, 56(3):483-497, 2010.

[28] S. Seth. Inequality, interactions, and human development. Journal of Human Development and Capabilities, 10(3):375-396, 2009.

[29] C. Herrero and A. Villar. On the comparison of group performance with categorical data. $P L o S$ ONE, 8(12):e84784, 2013. 